\title{
Applicability of mode-coupling theory to polyisobutylene: A molecular dynamics simulation study
}

\author{
Y. Khairy, ${ }^{1}$ F. Alvarez, ${ }^{1,2}$ A. Arbe, ${ }^{1}$ and J. Colmenero ${ }^{1,2,3, *}$ \\ ${ }^{1}$ Centro de Física de Materiales (CSIC-UPV/EHU) - Materials Physics Center (MPC), Paseo Manuel de Lardizabal 5 , \\ 20018 San Sebastián, Spain \\ ${ }^{2}$ Departamento de Física de Materiales UPVIEHU, Apartado 1072, 20080 San Sebastián, Spain \\ ${ }^{3}$ Donostia International Physics Center, Paseo Manuel de Lardizabal 4, 20018 San Sebastián, Spain
}

(Received 5 August 2013; published 4 October 2013)

\begin{abstract}
The applicability of Mode Coupling Theory (MCT) to the glass-forming polymer polyisobutylene (PIB) has been explored by using fully atomistic molecular dynamics simulations. MCT predictions for the so-called asymptotic regime have been successfully tested on the dynamic structure factor and the self-correlation function of PIB main-chain carbons calculated from the simulated cell. The factorization theorem and the time-temperature superposition principle are satisfied. A consistent fitting procedure of the simulation data to the MCT asymptotic power-laws predicted for the $\alpha$-relaxation regime has delivered the dynamic exponents of the theory-in particular, the exponent parameter $\lambda$-the critical non-ergodicity parameters, and the critical temperature $T_{c}$. The obtained values of $\lambda$ and $T_{c}$ agree, within the uncertainties involved in both studies, with those deduced from depolarized light scattering experiments [A. Kisliuk et al., J. Polym. Sci. Part B: Polym. Phys. 38, 2785 (2000)]. Both, $\lambda$ and $T_{c} / T_{g}$ values found for PIB are unusually large with respect to those commonly obtained in low molecular weight systems. Moreover, the high $T_{c} / T_{g}$ value is compatible with a certain correlation of this parameter with the fragility in Angell's classification. Conversely, the value of $\lambda$ is close to that reported for real polymers, simulated "realistic" polymers and simple polymer models with intramolecular barriers. In the framework of the MCT, such finding should be the signature of two different mechanisms for the glass-transition in real polymers: intermolecular packing and intramolecular barriers combined with chain connectivity.
\end{abstract}

DOI: 10.1103/PhysRevE.88.042302

\section{INTRODUCTION}

The aim of the Mode Coupling Theory (MCT) of the glasstransition is to describe the behavior of density correlators in deeply supercooled liquids or dense colloids [1-6]. In its original form, the MCT was formulated for simple liquids and it was numerically solved for rather simple systems as hard [7,8] and soft [9] spheres, a binary Lennard-Jones mixture [10] and simple aspherical molecules with an intramolecular orientational degree of freedom [11-14]. The application of MCT to the glass-transition of real systems is not trivial $[3,4]$. Experimental checks by neutron scattering-directly accessing density correlators-have been performed with model systems, such as, e.g., the molecular systems glycerol [15] and $o$-terphenyl [16]. In general, the results support MCT predictions at temperatures higher than the critical temperature of the theory $T_{c} \approx 1.2 T_{g}$ ( $T_{g}$ : glass-transition temperature), where most of the neutron scattering experiments have been carried out. Since they do not easily crystallize, another family of good candidates to check MCT are chemically simple polymers. In fact, neutron scattering results on 1,4-polybutadiene $(1,4-\mathrm{PB})[17,18]$ were in qualitative accordance with MCT. However, the applicability of this theory to the glass-transition of a polymer melt, where connectivity and internal degrees of freedom play major roles, remained as an open question.

Later, molecular dynamics (MD) simulations of a beadspring-type coarse-grained polymer model [19] could be consistently analyzed in terms of the predictions of MCT, motivating an extension of this theory [20] to reproduce the results

*juan.colmenero@ehu.es
PACS number(s): 64.70.pj, 61.20.Ja, 64.70.Q-, 61.41.+e

of such model polymer simulations. Regarding more realistic polymer models, MCT was applied to united-atom simulated polyethyelene (PE) [21] and 1,4-PB [22]. Furthermore, the derivation of Chong et al. [23] showed that the MCT equations for polymer melts become formally identical to the ones initially derived for simple liquids. Therefore, the asymptotic scaling laws deduced for such systems should also hold in the MCT for polymer melts. A phenomenological analysis of neutron scattering and/or computer simulation results on polymer melts is thus justified. In this light, fully atomistic simulated cells of 1,4-PB [24] and poly(vinyl methyl ether) (PVME) [25], properly validated by direct comparison with neutron scattering results, were used to check MCT asymptotic scaling laws. The predictions seem to be fulfilled in all cases, though high values of the exponent parameters are obtained as compared to those found for the bead-spring models [19]. The importance of the intramolecular barriers has been invoked to explain this effect $[24,26,27]$. Despite the progress made in this field, questions like the applicability of the theory to real polymer systems and the origin of the unusual values found for the critical exponents are still open.

From an experimental point of view, polyisobutylene (PIB, chemical formula: $\left.-\left[\mathrm{CH}_{2}-\mathrm{C}\left(\mathrm{CH}_{3}\right)_{2}\right]-{ }_{n}\right)$ is a very thoroughly investigated polymer by diverse techniques including spectroscopic techniques [28-31] as well as scattering methods [32-42]. It has the peculiarity of being one of the strongest polymers in Angell's terminology based on the concept of fragility [43]. In a couple of works some attempt to check MCT predictions with experimental results on PIB were made. One of them was the neutron spin echo investigation on collective and self-motions by Farago et al. [39]. In that work, special emphasis was made in the exploration of the so-called intermediate length scales regime. Intermediate 
scales means the region of lengths larger than intermolecular distances but smaller than the hydrodynamic range. The MCT prediction regarding the momentum transfer $(Q)$ and temperature dependence of the collective relaxation times was checked in the $Q$-range around the first static structure peak and covering the intermediate scales. The authors found discrepancies between experiments and theory, that in fact are not surprising since the MCT is not expected to apply in such intermediate $Q$-range. On the other hand, Kisliuk et al. [37] successfully described depolarized light scattering spectra at temperatures above $\approx 280 \mathrm{~K}$ using power laws as predicted by the theory. From the exponents of these laws the values of the exponents $a$ and $b$ (see later) were deduced: $a=0.5-0.6 ; b=0.34-0.4$. The values for the $a$-parameter were inconsistent with the MCT, but this observation was disregarded as an intrinsic problem of the theory. The argument was that such a quantitative disagreement had previously been observed for low molecular weight systems and also for poly(propylene glycol) (PPG) and was ascribed to a strong vibrational contribution (the Boson peak) to the fast process $[44,45]$. In Ref. [46] it was shown however that such a disagreement is overcome properly including the vibrational contribution. We note that the Boson peak in PIB is particularly prominent with respect to other polymeric materials [32,33]. Regarding the critical temperature of the MCT $T_{c}$, the results of Ref. [37] indicated a rather large value with respect to the experimental glass-transition, $T_{c} / T_{g} \approx 1.35$.

In this work we apply a phenomenological MCT analysis to MD-simulation results on PIB, in an analogous way as previously performed in the cases of 1,4-PB [24] and PVME [25]. We first check the factorization theorem and the timetemperature superposition principle. Thereafter we present a consistent fitting procedure of the simulation data to the MCT asymptotic power laws predicted for the $\alpha$-relaxation regime, in order to obtain information about the dynamic exponents of the theory, the critical nonergodicity parameters and the critical temperature. Finally, we discuss the results in comparison with those reported for other glass-forming systems including polymers.

\section{MOLECULAR DYNAMICS SIMULATIONS}

Fully atomistic molecular dynamics simulations were carried out using the COMPASS force field. Based on the PCFF force field, the COMPASS was developed using an hybrid approach consisting of $a b$ initio parametrization and empirical optimization method. The former method was used to derive the force field parameters, while the latter yielded a good agreement between calculation and experiments. The force field functional forms are characterized by two different kind of functions: the valence terms, which include diagonal and off-diagonal cross-coupling terms, and the nonbond interaction terms. The valence terms represent internal coordinates of bond, angle, torsion angle and out-of-plane angle, the cross-coupling terms include combinations of two or three internal coordinates. The nonbond interactions include a Lennard-Jones 9-6 function for the Van der Waals terms and a Coulombic function for electrostatic interactions. Details of the analytical expression of the functional form are given in Refs. [47-49].
The initial configuration of the simulated system was built by means of the MATERIALS STUDIO 5.0 Amorphous Cell builder [50]. A cubic cell containing 20 PIB chains of 70 monomers $\left(M_{w}=3923 \mathrm{~g} / \mathrm{mol}\right.$, i.e., smaller than the entanglement mass $M_{e} \approx 7000 \mathrm{~g} / \mathrm{mol}$ [51], and a total number of atoms $N=16840$ ) was constructed at a rather high temperature of $500 \mathrm{~K}$ (experimental $T_{g} \sim 200 \mathrm{~K}$ ), under periodic boundary conditions. By means of NPT dynamic runs (i.e., keeping constant the number of atoms, pressure, and temperature) a value of the density of $\rho=0.8109 \mathrm{~g} / \mathrm{cm}^{3}$ was determined. This value is in excellent agreement with the literature data $\rho_{\exp }=0.8102 \mathrm{~g} / \mathrm{cm}^{3}$ [52]. Such a density leads to a cell dimension of $54.12 \AA$ for the cubic side. In order to minimize the obtained energy structure, the Polak-Ribiere conjugated gradients method was used. After the minimization, the system was dynamically equilibrated by a successive $N V T$ run (i.e., keeping constant the number of atoms, volume, and temperature) of $1 \mathrm{~ns}$. The system obtained in such way was used as a starting point for collecting data every $0.01 \mathrm{ps}$ during a run of $1 \mathrm{~ns}$. The production simulations were carried out in the $N V T$ ensemble and the velocity-Verlet algorithm with a time step of $1 \mathrm{fs}$ was used as the integration method. To control the temperature, instead of a real temperature-bath coupling (Nose-Hoover or Berendsen thermostat) a velocity scaling procedure with a wide temperature window of $10 \mathrm{~K}$ was followed. Under these conditions, greater temperature fluctuations are allowed but the trajectory is disturbed less. It has been checked that, by following this simple procedure in similar polymeric systems, the results are similar to those obtained with an $N V E$ ensemble (where the number of atoms, volume, and energy are kept constant), which has the proper Newtonian dynamics [53]. After the first $1 \mathrm{~ns}$ run, successive runs of $4 \mathrm{~ns}$ collecting data every $0.5 \mathrm{ps}$ were carried out in order to reach a $100 \mathrm{~ns}$ dynamics. This cell was used to yield simulated systems at the lower temperatures of $470,390,365,335$, and $320 \mathrm{~K}$. The new cells were obtained scaling the temperature and then were equilibrated running three dynamics of $1 \mathrm{~ns}$. Once the equilibrium density was reached for each temperature, the same protocol of the first simulated sample was followed to carry out a $100 \mathrm{~ns}$ dynamics. The densities of the equilibrated cells were found to be $\rho(470 K)=0.8264 \mathrm{~g} / \mathrm{cm}^{3}, \rho(390 K)=0.8688 \mathrm{~g} / \mathrm{cm}^{3}$, $\rho(365 K)=0.8820 \mathrm{~g} / \mathrm{cm}^{3}, \rho(335 K)=0.8977 \mathrm{~g} / \mathrm{cm}^{3}$, and $\rho(320 K)=0.9055 \mathrm{~g} / \mathrm{cm}^{3}$.

The validation of the simulated cell has been thoroughly carried out $[54,55]$ by direct comparison of magnitudes computed from the simulation and measured by neutron scattering on protonated and deuterated samples in earlier works $[32,39,42]$. The agreement found is excellent regarding both static and dynamic features. The latter comprise selfatomic (hydrogen) motions and collective motions.

\section{MODE COUPLING THEORY ANALYSIS OF PIB}

One of the central predictions of the MCT is the existence of a critical temperature $T_{c}$ where the system shows structural arrest driven by the mutual blocking of each particle by its neighbors. This ergodic-to-nonergodic transition is called the idealized glass-transition of the MCT. The critical non-ergodic parameter, $f_{Q}^{c}$, for wave vector $Q$, is defined as the long-time 
limit of the normalized density-density correlator $S(Q, t)$ at $T_{c}$. A similar definition is given for the self-non-ergodic critical parameter, $f_{Q}^{s c}$, corresponding to the normalized self-correlation function $F_{\text {self }}(Q, t) . f_{Q}^{c}\left(f_{Q}^{s c}\right)$ has also the meaning of the plateau hight in the two-step relaxation of $S(Q, t)\left[F_{\text {self }}(Q, t)\right]$ and thereby it provides a measure of the localization length, i.e., the dimension of the cage in which the particles are trapped.

The existence of $T_{c}$ allows to introduce the separation parameter $|\epsilon|=\left(T-T_{c}\right) / T_{c}$, which quantifies the relative distance of the temperature of the system to the critical temperature (it can also be defined for density or any other control parameter). As it is nicely described in Ref. [3], the essence of this transition scenario can be understood by asymptotic solutions of the MCT equations for times longer than that associated to the frequency of the normalsystem excitations and for temperatures (control parameters) approaching the critical value. These general laws are known as the "universalities" or asymptotic formulas of the MCT. There are two different relevant regimes for these asymptotic solutions. The first deals with the near-plateau-relaxation phenomena, i.e., with the processes referred to as $\beta$-relaxation in the original MCT literature. The second scaling law regime deals with the below-plateau decay processes, which are also called $\alpha$-processes.

The separation parameter determines two relevant time scales of the ideal MCT for these two regions: the $\beta$-relaxation time, $t_{\sigma}=t_{o}|\sigma|^{-1 /(2 a)}$ and the $\alpha$-relaxation time $\tau_{\sigma}=t_{o} B^{-1 / b}|\sigma|^{-\gamma}$, where $\sigma=C_{\sigma}|\epsilon|$ and $t_{o}$ denotes a microscopic time scale associated to the frequency of the normal system excitations. The constants $C_{\sigma}$ and $B$ are system dependent and $Q$ - and $|\epsilon|$-independent. The exponent $\gamma$ is defined as

$$
\gamma=\frac{1}{2 a}+\frac{1}{2 b}
$$

and the $b$-exponent $(0<b<1)$ is called the "von Schweidler" exponent. On the other hand, the exponents $a$ and $b$ are not independent of each other. They are related by the "exponent parameter $\lambda "(\lambda \leqslant 1)$ as

$$
\lambda=\frac{\Gamma^{2}(1+b)}{\Gamma(1+2 b)}=\frac{\Gamma^{2}(1-a)}{\Gamma(1-2 a)},
$$

where $\Gamma$ is Euler's Gamma function. This exponent parameter does not depend on temperature. It is determined by the equilibrium properties of the system at $T_{c}$.

The so-called "phenomenological MCT analysis" of experimental and MD simulations results in general is based on the asymptotic laws mentioned above. This has been the procedure previously applied to atomistic MD simulations of several polymers [24,25] and to simulations of different coarse-grained models of polymers [19,21,22,27]. This is also the procedure we are following here. It will be applied to two correlation functions $\phi_{Q}(t)$ revealing collective and self motions: (i) the dynamic structure factor (density-density correlator) $S(Q, t)$ :

$$
S(Q, t)=\frac{\langle\rho(Q, t) \rho(-Q, 0)\rangle}{\langle\rho(Q, 0) \rho(-Q, 0)\rangle},
$$

where the density correlator is $\rho(Q, t)=\sum_{i} \exp \left[i \vec{Q} \vec{r}_{i}(t)\right]$ and $\vec{r}_{i}(t)$ denotes the position of atom $i$ in the system; and (ii) the self-correlation function

$$
F_{\text {self }}(Q, t)=\frac{1}{N}\left\langle\sum_{i} e^{i \vec{Q}\left[\vec{r}_{i}(t)-\vec{r}_{i}(0)\right]}\right\rangle .
$$

As representative subensemble of atoms to apply the theory for $F_{\text {self }}(Q, t)$ we have chosen the main-chain carbons. In this way the particularities associated to the additional motions of methyl groups are avoided. In any case, in the next section the influence of this kind of dynamics will be discussed. This means, $F_{\text {self }}(Q, t)$ has been calculated here restricting the sum in Eq. (4) to the coordinates of main-chain carbons.

In practice, the temperature and time intervals where the asymptotic laws can be applied are not known a priori. The predictions are expected to hold close to the critical temperature $T_{c}$ for small values of the separation parameter $\epsilon$. We can define a range of temperatures $T_{c} \lesssim T \lesssim T_{\max }$ where the asymptotic laws can be applied to fit the simulation data. As mentioned above, a priori the value of $T_{c}$ is unknown. A first guess would be $T_{c} \approx 1.2 T_{g}$, as it is usually found in the literature. For PIB the experimental glass-transition is $205 \mathrm{~K}$, leading to a first estimation of $T_{c} \approx 250 \mathrm{~K}$. Thus, all the temperatures of the simulations are expected to be above the critical one. In the other extreme, $T_{\max }$ is some high temperature for which the density correlator still exhibits a two-step relaxation. This could be around $470 \mathrm{~K}$. The good choice of time and temperature intervals will be justified at the end by the internal consistency of the results obtained when the theory is applied.

\section{A. First universality: Factorization theorem}

A MCT prediction that can be tested without invoking fits or knowledge of the values of the critical parameters is the so-called factorization theorem or first universality in the $\beta$-regime. Mathematically, the $\beta$-regime is defined as the time window $t_{o} \ll t \ll \tau_{\sigma}$ in which $\left|\Phi_{Q}(t)-f_{Q}^{c}\right| \ll 1$ for the correlator $\phi_{Q}(t)$. In this window, MCT predicts that for $T \rightarrow T_{c}^{+}$the correlator $\phi_{Q}(t)$ follows the asymptotic law: $\phi_{Q}(t)=f_{Q}^{c}+h_{Q} G(t)$. It means that deviations from $f_{Q}^{c}$ in this time regime factorize in a $Q$-dependent term (the critical amplitude $\left.h_{Q}\right)$ and a $t$-dependent term $G(t)=\sqrt{\sigma} g\left(t / t_{\sigma}\right)$. The function $g\left(t / t_{\sigma}\right)$ is called the " $\beta$-correlator" and it should be identical for all $\phi_{Q}(t)$. It can be shown that $g\left(t / t_{\sigma}\right)$ has the following asymptotic forms: $g\left(t / t_{\sigma}\right)=\left(t / t_{\sigma}\right)^{-a}$ for $t \ll t_{\sigma}$ and $g\left(t / t_{\sigma}\right)=-B\left(t / t_{\sigma}\right)^{b}$ for $t_{\sigma} \ll t \ll \tau_{\sigma}$. This last functional form is called "von Schweidler law". If the factorization theorem works, the ratio

$$
R_{Q}(t)=\frac{\phi_{Q}(t)-\phi_{Q}\left(t^{\prime}\right)}{\phi_{Q}\left(t^{\prime \prime}\right)-\phi_{Q}\left(t^{\prime}\right)}=\frac{G(t)-G\left(t^{\prime}\right)}{G\left(t^{\prime \prime}\right)-G\left(t^{\prime}\right)}
$$

would depend on the fixed times $t^{\prime}$ and $t^{\prime \prime}$ and on the temperature, but must be independent of $Q$ and the correlator considered. Figure 1 shows that at $335 \mathrm{~K}$ the superposition of $R_{Q}(t)$ calculated at different $Q$-values for both, self- and collective correlators, is almost perfect in the region $1 \mathrm{ps} \lesssim t \lesssim$ $0.2 \mathrm{~ns}$, splitting at both shorter and longer times. Moreover, Fig. 2 demonstrates that the results for self- and density-density correlators collapse into a single master-curve in this time interval. Thus, there is an intermediate time window of more 


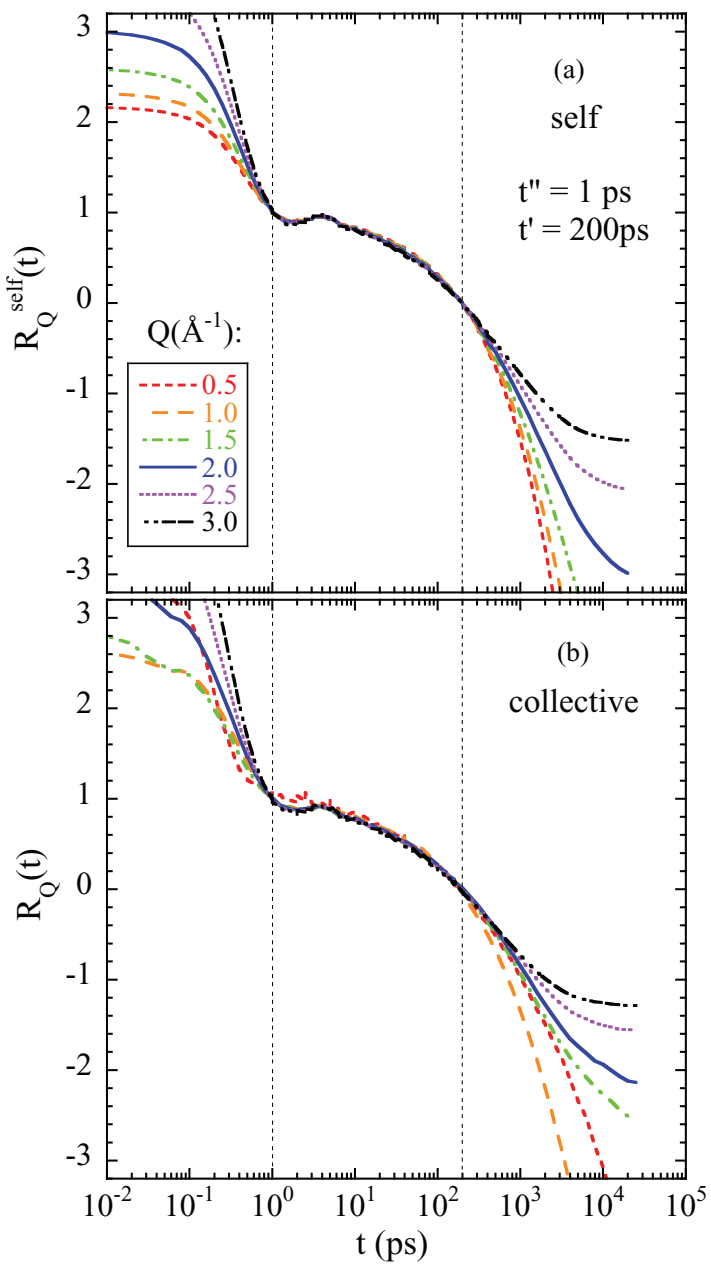

FIG. 1. (Color online) Test of the factorization theorem applied to the self-correlation function of the main-chain carbons $F_{\text {self }}(Q, t)$ (a) and to the dynamic structure factor $S(Q, t)$ (b) at $335 \mathrm{~K}$ and the $Q$-values indicated in (a). $t^{\prime}$ and $t^{\prime \prime}$ (vertical dotted lines) represent the limits of the $\beta$-window.

than two decades in which the factorization theorem holds for $\mathrm{PIB}$ at this temperature-the MCT $\beta$-relaxation regime.

In the case of MD-simulation data, the factorization theorem can also be checked in real space, using the van Hove correlation functions [19]. It reads $G(r, t)=F(r)+H(r) G(t)$. In the $\beta$-regime, the ratio

$$
R(r, t)=\frac{G(r, t)-G\left(r, t^{\prime}\right)}{G\left(r^{\prime}, t\right)-G\left(r^{\prime}, t^{\prime}\right)}=\frac{H(r)}{H\left(r^{\prime}\right)}
$$

should be independent of time and inform us about the length scales involved in this process. In Fig. 3 we can see the good master curves obtained considering times in the interval between 1 and 100 ps for the self-part of the van Hove correlation function. This time regime is within that determined for the $\beta$-window from the analysis in the reciprocal space. At faster and longer times failure of the superposition is observed. Figure 3 shows that the motions of main chain carbons are limited to displacements smaller than $\approx 3.5 \AA$. This value is clearly smaller than the average intermolecular distance in this polymer, that can be estimated in the Bragg approximation from the first peak position

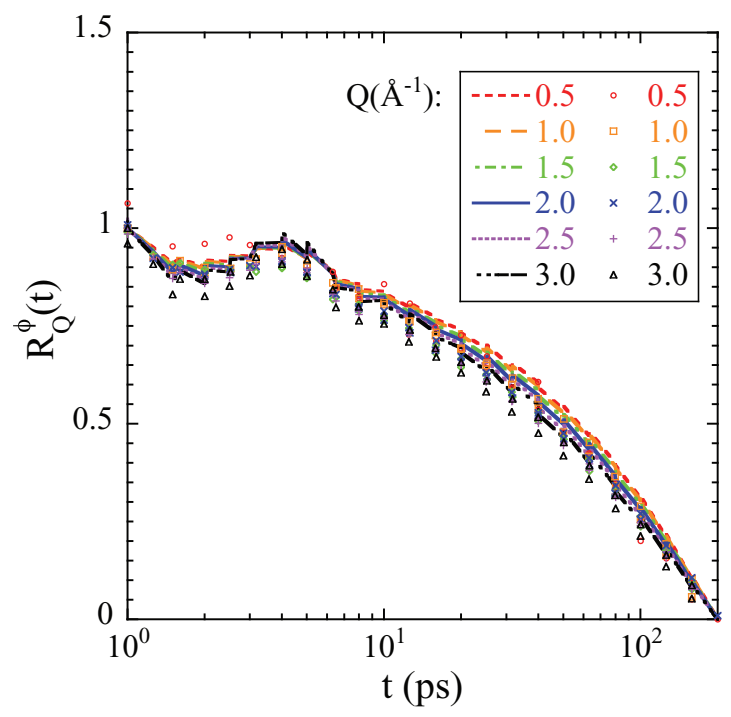

FIG. 2. (Color online) Time dependence of $R_{Q}(t)$ for both self(lines) and collective (points) correlators at $335 \mathrm{~K}$ for the $Q$-values indicated.

observed in the simulated structure factor $\left(Q_{1 \max }=1 \AA^{-1}\right)$. We obtain $d_{\text {chain }} \approx 2 \pi / Q_{1 \max }=6.3 \AA$. At small distances, the function $R(r, t)$ shows positive values with a peak at about $0.5 \AA$, vanishes at $r_{o}=0.92 \AA$ and displays a negative peak at longer distances. We note that the function $H(r)$ determines the rate at which the van Hove correlation relaxes [19]:

$$
\frac{\partial G(r, t)}{\partial t} \propto-H(r) \text {. }
$$

This implies that in the $\beta$-regime the probability for atomic displacements of size $r$ decreases most where $H(r)$ is largest $(r \approx 0.5 \AA)$, and increases most where $H(r)$ is smallest $(r \approx 1.3 \AA)$. This last distance is much smaller than $d_{\text {chain }}$; therefore, attempts to overcome distances of the order of the

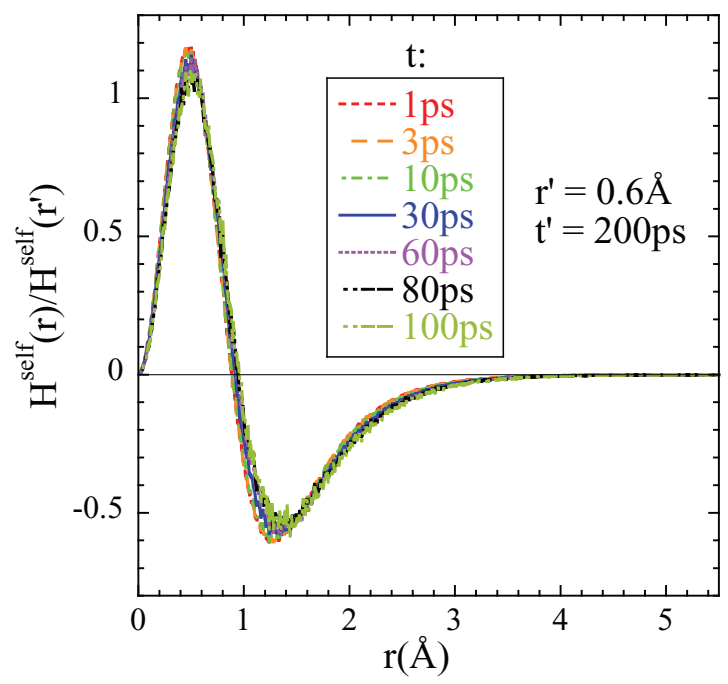

FIG. 3. (Color online) Factorization theorem in the real space for the self-part of the Van Hove scattering function evaluated for the main-chain carbons. The theorem has been applied to simulated data at $335 \mathrm{~K}$ at the different indicated times. The value of $r^{\prime}$ has been chosen such that the denominator in Eq. (6) is maximized. 
intermolecular separation are partially reflected back and the atom returns close to its initial position $[19,56]$.

\section{B. Second universality: Time-temperature superposition principle in the $\alpha$-regime}

As we have mentioned above, the term $\alpha$-regime refers to the decay of a given correlator $\phi_{Q}(t)$ from the plateau value $\left(f_{Q}^{c}, f_{Q}^{s c}\right)$ to zero. This decay occurs for times longer than the $\beta$-relaxation time $t_{\sigma}$, thus overlapping with the $\beta$ process for $t_{\sigma} \leqslant t \leqslant \tau_{\sigma}$. Ideal MCT predicts that the $\alpha$-process satisfies a time-temperature superposition principle (TTSP) for $T \rightarrow T_{c}^{+}$. This means that the correlator $\phi_{Q}(t)$ at temperature $T$ can be written as $\phi_{Q}(t, T)=\tilde{\phi}_{Q}\left(t / \tau_{\sigma}\right)$, where $\tilde{\phi}_{Q}$ is a $T$-independent master function. The shape of $\tilde{\phi}_{Q}$ is often well described by the empirical Kohlrausch-Williams-Watts (KWW) expression

$$
\phi_{Q}^{K W W}(t) \propto \exp \left[-\left(\frac{t}{\tau_{Q}^{K W W}}\right)^{\beta}\right] .
$$

We note however that the KWW-function does not come out as an analytical solution of the MCT equations.

In Fig. 4 the TTSP is checked for self- (a) and collective (b) dynamics of PIB at the first structure factor $Q$-value $Q_{1 \max }$. The timescale chosen to superimpose the data is that where the correlation function takes the value 0.2 . For both correlators we observe a very good superposition in the temperature range $335 \mathrm{~K} \leqslant T \leqslant 470 \mathrm{~K}$ that extends over more than two decades in the long-time region. At the highest temperature $(500 \mathrm{~K})$ the two-step feature of the dynamic structure factor is not so clear and, as above anticipated, the MCT predictions start failing. Also deviations from a perfect superposition could be envisaged for $320 \mathrm{~K}$ in Fig. 4(a). The deviations observed from the ideal MCT behavior are attributed to the presence of additional relaxation mechanisms ignored by the ideal version of the theory, the so-called "hopping processes". These processes become dominant on approaching the critical temperature from above and restore ergodicity below $T_{c}$.

Thus from the time-temperature superposition principle we can deduce that the interval in which MCT is expected to hold in our PIB simulations is $335 \mathrm{~K} \lesssim T \lesssim 470 \mathrm{~K}$.

\section{MCT asymptotic power-laws}

Now we obtain the values of the critical parameters for PIB and test the consistency of other MCT predictions by studying the behavior of parameters that can easily be obtained from a phenomenological data analysis. As it has been mentioned above, the KWW function [Eq. (8)] is not an analytical solution of the MCT for the $\alpha$-decay in general. However, there is a special case, the limit of large $Q(Q \rightarrow \infty)$. In this limit it was proved [57] that $\lim _{Q \rightarrow \infty} \tilde{\bar{\phi}}_{Q}(t) \sim \phi_{Q}^{\mathrm{KWW}}(t)$ with $\beta=b$ and $\tau_{Q}^{\mathrm{KWW}} \propto Q^{-1 / b}$. Therefore, this provides a simple procedure to obtain a value for the von Schweidler exponent $b$. In order to do that, we have fitted the last stage of the decay of the correlation functions by a KWW expression. From these fits, the $Q$ - and $T$-dependent shape parameter $\beta$ was obtained. The resulting values for two temperatures in the interval where MCT is expected to hold are shown in Fig. 5. For both correlation functions considered, the KWW exponent tends to an average

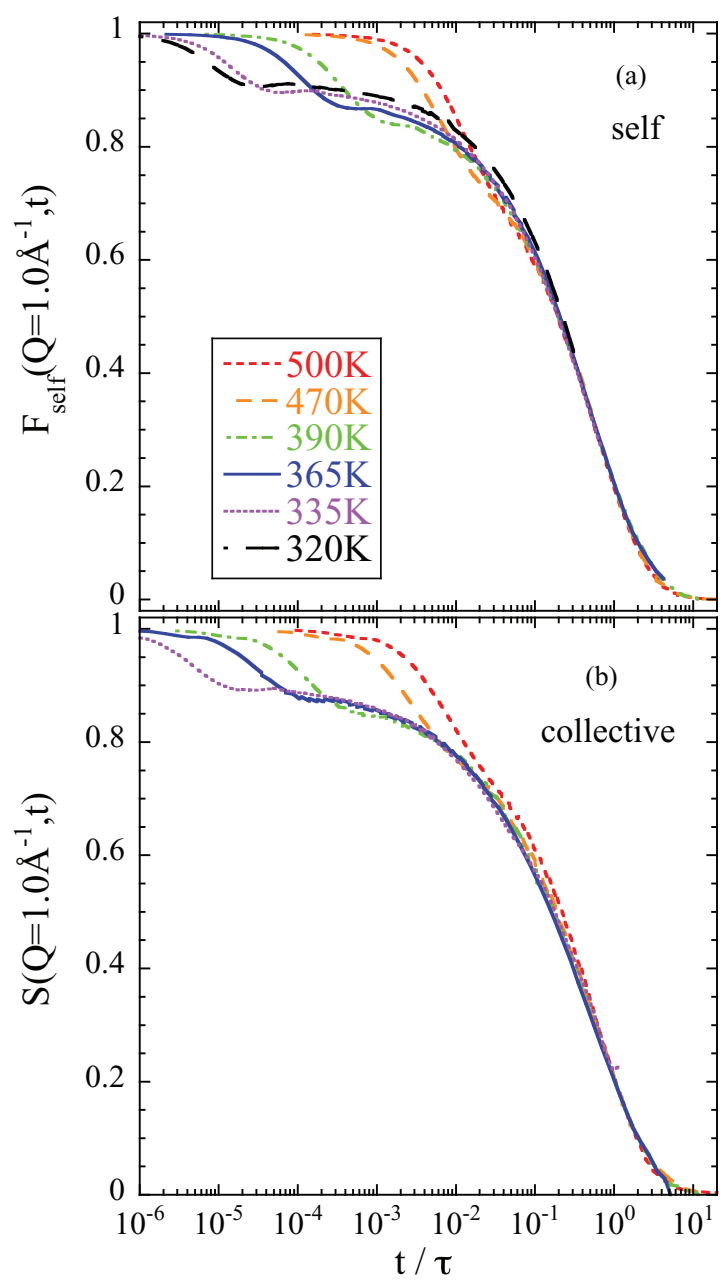

FIG. 4. (Color online) Test of the time-temperature superposition principle for $\phi_{Q}(t)$ at the first peak structure factor $Q$-value $\left(Q_{\max }=\right.$ $\left.1 \AA^{-1}\right)$ : self-correlation function of main-chain carbons $F_{\text {self }}(Q, t)$ (a) and dynamic structure factor $S(Q, t)$ (b) at the indicated temperatures. The $S(Q, t)$ data at $320 \mathrm{~K}$ are not included because the function does not decay down to 0.2 in the investigated time window.

value of about 0.32 for high $Q$. This value was thus chosen for the $b$ exponent, though there is of course an uncertainty involved in this determination (we could not discard $b$-values within the range $0.28-0.38$ ).

The simple KWW description used to derive the value of the $b$-exponent also provides information to check the other prediction of MCT for large $Q: \tau_{O}^{\mathrm{KWW}} \propto Q^{-1 / b}$. As can be seen in Fig. 6 for $335 \mathrm{~K}$, this prediction is also verified in a good approximation for PIB.

Considering $b=0.32$, from the MCT relations given by Eqs. (2) and (1) values of $a=0.22, \lambda=0.89$ and $\gamma=3.84$ are obtained.

On the other hand, the initial part of the $\alpha$-process (the von Schweidler regime) is given by the power-law expansion

$$
\phi_{Q}(t)=f_{Q}-H_{1 Q} t^{b}+H_{2 Q} t^{2 b}+\ldots
$$

which extends the von Schweidler law. Then, a description of the decay from the plateau by means of Eq. (9) should be possible with the $b$-value above obtained and with a $T$-independent critical nonergodicity parameter $f_{Q}^{c}\left(f_{Q}^{s c}\right)$. We 


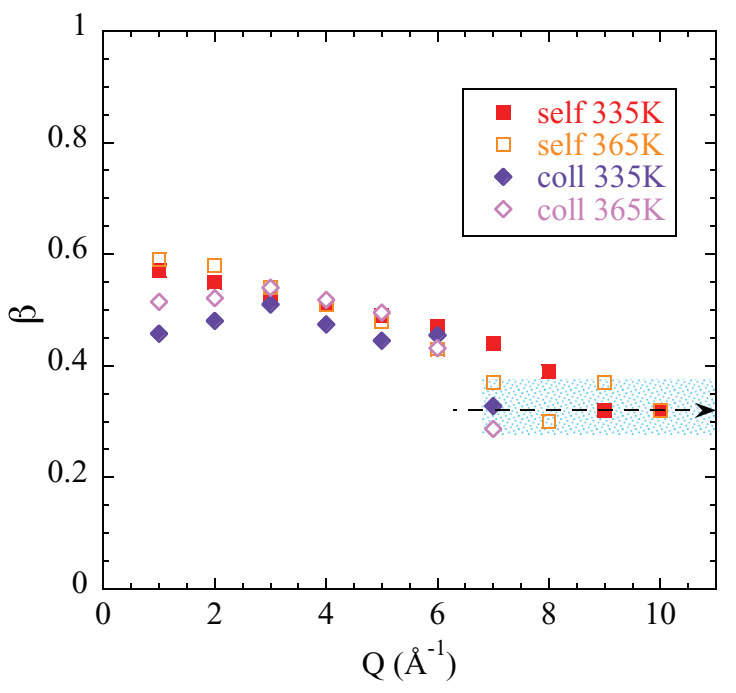

FIG. 5. (Color online) $Q$-dependence of the stretching parameter $\beta$ evaluated at $335 \mathrm{~K}$ (solid) and $365 \mathrm{~K}$ (empty) of the dynamic structure factor $S(Q, t)$ (diamonds) and the self-correlation function of main-chain carbons $F_{\text {self }}(Q, t)$ (squares). The arrow marks the average asymptotic value, and the shadowed area indicates the uncertainty range.

have first considered the correlation functions at different temperatures. For each $Q$-value, all the curves can be well described with a single value of $f_{Q}^{c}\left(f_{Q}^{s c}\right)$. This holds for self-correlations (see Fig. 7) as well as for the dynamic structure factor (see Fig. 8). The fact that both the incoherent scattering function and the density-density correlator can be reasonably described by Eq. (9) with $T$-independent values of the nonergodicity parameter supports again the applicability of MCT predictions to this polymer data.

The values of the nonergodicity parameters used in the fits are displayed in Fig. 9. Those corresponding to the selfcorrelation function $f_{Q}^{s c}$ can be described, within the uncertainties, by the Gaussian approximation $f_{Q}^{s c}=\exp \left(-Q^{2} r_{s c}^{2}\right)$,

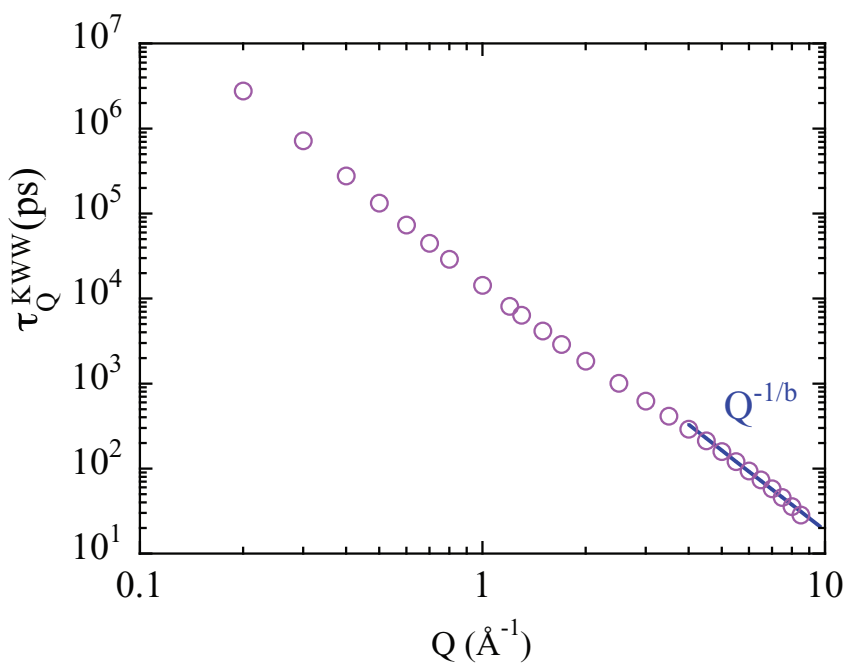

FIG. 6. (Color online) $Q$-dependence of the characteristic KWW time of the self-correlation function of main-chain carbons at $335 \mathrm{~K}$. The line shows the MCT prediction of the asymptotic dependence of this time for $Q \rightarrow \infty$, namely $\tau_{Q}^{\mathrm{KWW}} \propto Q^{-1 / b}$.

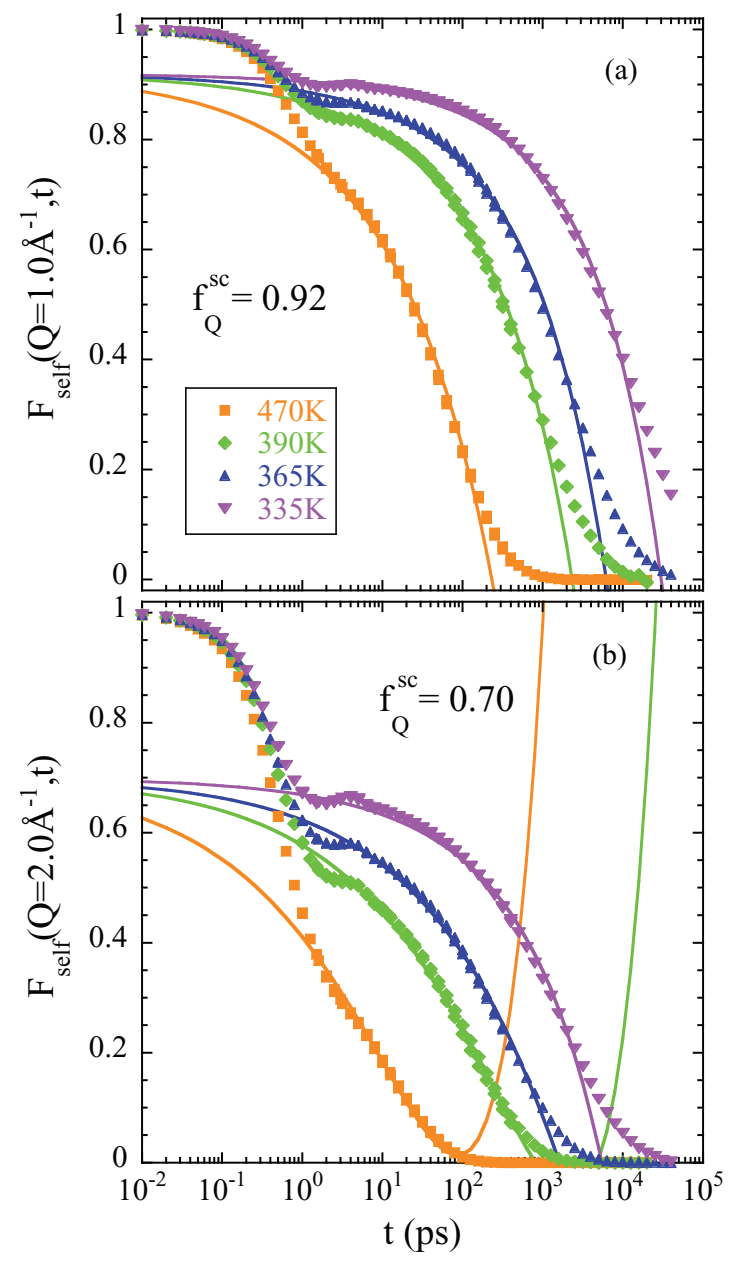

FIG. 7. (Color online) Fit of the MCT von Schweidler regime of $F_{\text {self }}(Q, t)$ at $Q=1.0 \AA^{-1}$ (a) and $Q=2.0 \AA^{-1}$ (b) at the temperatures indicated in (a). For each $Q$-value, all the curves are described with a single value of the nonergodicity parameter $f_{Q}^{s c}$.

from which a localization length of $r_{s c}=0.30 \AA$ is deduced. Conversely, for $S(Q, t)$ the nonergodicity parameter is modulated by the static structure factor, according with MCT.

Finally we can determine the value of the critical temperature $T_{c}$. This can be done by applying two independent MCT asymptotic power-laws. The first one is that involving the $H_{1 Q}$ parameters in Eq. (9), which should fulfill the relation

$$
H_{1 Q} \propto\left|T-T_{c}\right|^{\gamma b} .
$$

Applying this equation to the results obtained from the analysis of both $F_{\text {self }}(Q, t)$ and $S(Q, t)$ a value of $T_{c} \approx 315 \mathrm{~K}$ is deduced. The second MCT law is that giving the temperature dependence of the characteristic time for the structural $\alpha$-relaxation. In the framework of the MCT, the way this characteristic time approaches the critical temperature $T_{c}$ is determined by the $\gamma$-parameter as

$$
\tau \propto\left|T-T_{c}\right|^{-\gamma} .
$$

Here, in order to be sure that we avoid any contribution from the $\beta$-regime, we have considered as characteristic time of the $\alpha$-process, the time $\tau$ at which the corresponding correlation 


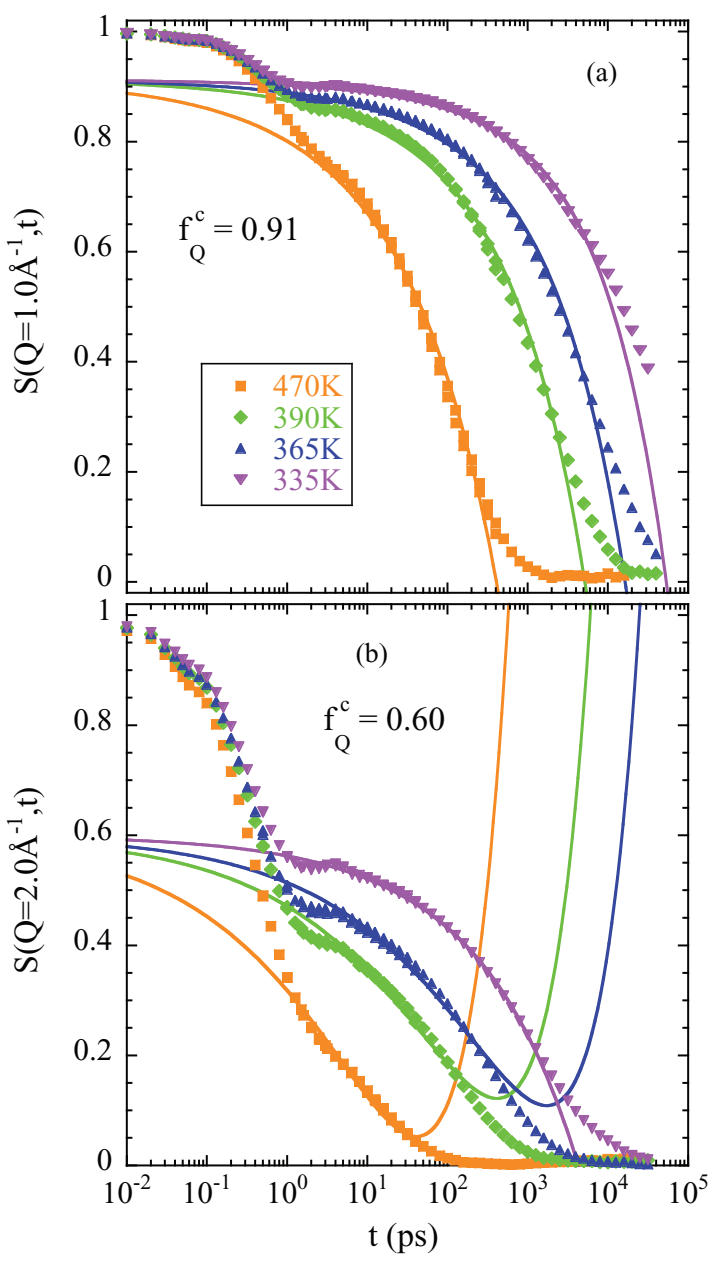

FIG. 8. (Color online) Fit of the MCT von Schweidler regime of the $S(Q, t)$ at $Q=1.0 \AA^{-1}$ (a) and $Q=2.0 \AA^{-1}$ (b) at the temperatures indicated in (a). For each $Q$-value, all the curves are described with a single value of the nonergodicity parameter $f_{Q}^{c}$.

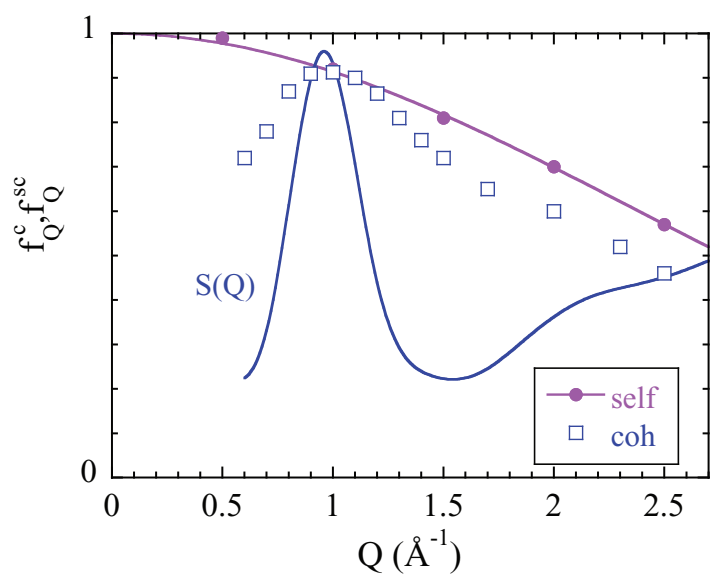

FIG. 9. (Color online) $Q$-dependence of the nonergodicity parameter calculated for the dynamic structure factor $S(Q, t)$ and the self-correlation function of main-chain carbons $F_{\text {self }}(Q, t)$. The solid line through the latter represents the fit with the expression $f_{Q}^{s c}=\exp \left(-Q^{2} r_{s c}^{2}\right)$, with $r_{s c}=0.30 \AA$. The static structure factor at an intermediate temperature $(365 \mathrm{~K})$ is also shown for comparison.

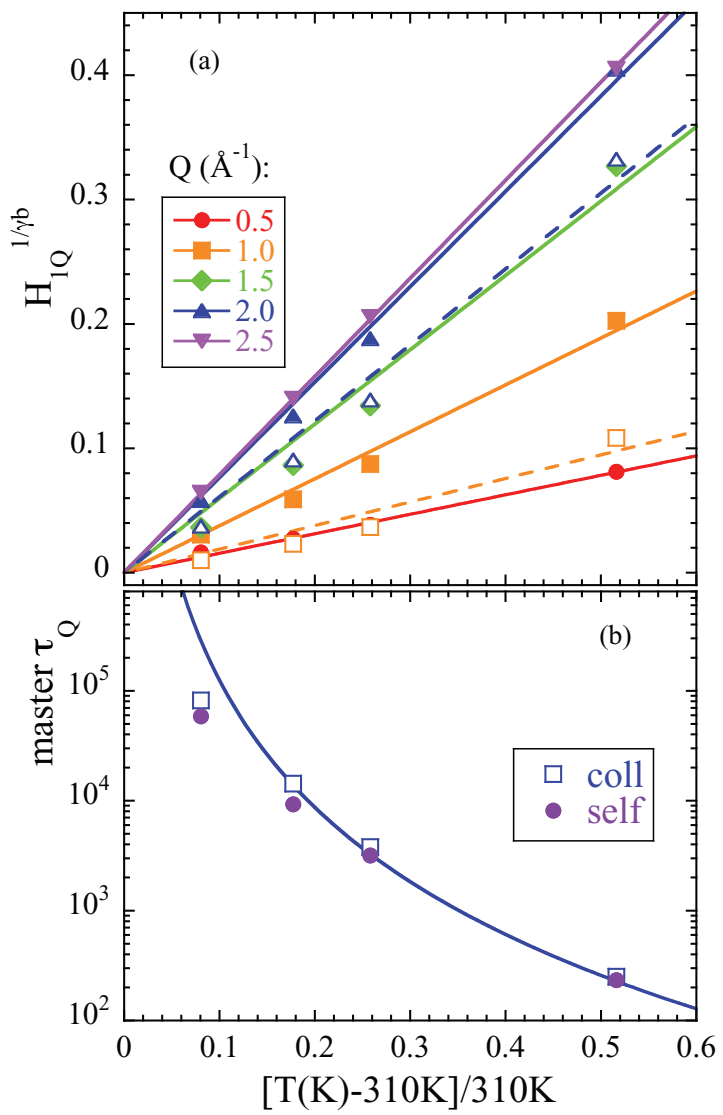

FIG. 10. (Color online) (a) Temperature dependence of the $H_{1 Q}$-parameter of the MCT von Schweidler expansion of the selfcorrelation function of the main-chain carbons $F_{\text {self }}(Q, t)$ (filled symbols) and dynamic structure factor $S(Q, t)$ (empty symbols) at different $Q$-values. Lines show the fit with expression (10). (b) Test of the MCT prediction for the temperature dependence of the $\alpha$-relaxation characteristic time [Eq. (11)]. The times have been defined as those where the normalized correlation functions take the value 0.2 . The data correspond to $F_{\text {self }}\left(Q_{1 \max }=1 \AA^{-1}, t\right)$ and $S\left(Q_{1 \max }=1 \AA^{-1}, t\right)$ scaled into a master curve (the selfcharacteristic times have been multiplied by 2 to match the collective ones).

function at $Q_{1 \max }=1 \AA^{-1}$ reaches the value 0.2 . We note that the superposition principle above discussed implies that the $\alpha$-relaxation time defined in this way is proportional to $\tau_{\sigma}$. By considering the $\tau$-values so obtained and the $\gamma$-value previously determined, a fit of Eq. (11) to the results in the range $335 \mathrm{~K} \leqslant T \leqslant 470 \mathrm{~K}$ reveals $T_{c} \approx 293 \mathrm{~K}$, i.e., about $20 \mathrm{~K}$ lower than the value determined through Eq. (10). A similar situation was also reported in the case of a MCT analysis of MD simulations of 1,4-PB [24]. As in that case, the two values of $T_{c}$ independently obtained here differ by only $6 \%$, which would be compatible within the error bars. In fact, Fig. 10 shows that choosing an intermediate value of $310 \mathrm{~K}$ for $T_{c}$ an overall satisfactory description of both sets of parameters $H_{1 Q}$ and $\tau$ in terms of MCT predictions Eqs. (10) and (11) is achieved. The deviations of $\tau$ from Eq. (11) at the lowest temperature considered for the MCT analysis (335 K) could be justified invoking already at this temperature some slight influence of the above-mentioned hopping processes. Such contribution 
TABLE I. Values of the MCT critical exponents for different systems

\begin{tabular}{|c|c|c|c|c|c|}
\hline System & $a$ & $b$ & $\gamma$ & $\lambda$ & Ref. \\
\hline Hard spheres & 0.31 & 0.58 & 2.5 & 0.74 & [14] \\
\hline o-terphenyl (MD) & 0.30 & 0.54 & 2.6 & 0.76 & {$[60]$} \\
\hline o-terphenyl (exp.) & 0.295 & 0.53 & 2.6 & 0.77 & {$[16,61]$} \\
\hline Silica (MD) & 0.32 & 0.62 & 2.3 & 0.71 & [62] \\
\hline Glycerol (exp.) & 0.32 & 0.61 & 2.3 & 0.72 & [15] \\
\hline Water (MD) & 0.29 & 0.51 & 2.7 & 0.78 & {$[63]$} \\
\hline Bead-spring polymer (MD) & 0.35 & 0.75 & 2.1 & 0.63 & [19] \\
\hline Bead-spring polymer (MCT) & 0.32 & 0.60 & 2.4 & 0.72 & [19] \\
\hline Bead-spring polymer (strong barriers, MD) & 0.22 & 0.33 & 3.8 & 0.89 & {$[27]$} \\
\hline Polyethylene (united atom) & 0.27 & 0.46 & 2.9 & 0.81 & [21] \\
\hline 1,4-Polybutadiene (exp.) & & $0.30-0.37$ & 3.2 & $0.86-0.90$ & [18] \\
\hline 1,4-Polybutadiene (united atom) & 0.21 & 0.30 & 4.1 & 0.90 & [22] \\
\hline 1,4-Polybutadiene (fully atomistic) & 0.18 & 0.24 & 4.9 & 0.93 & [24] \\
\hline Poly(vinyl methyl ether) (fully atomistic) & 0.23 & 0.35 & 3.6 & 0.87 & {$[25]$} \\
\hline Polyisobutylene (fully atomistic) & 0.22 & 0.32 & 3.8 & 0.89 & [this work] \\
\hline Polyisobutylene (exp.) & $0.5-0.6$ & $0.35-0.6$ & & & {$[37]$} \\
\hline Polystyrene (exp.) & $0.35-0.4$ & $0.3-0.35$ & & & [37] \\
\hline Poly(propylene glycol) (exp.) & $0.23 \pm 0.05$ & $0.36 \pm 0.02$ & $3.6 \pm 0.2$ & $0.87 \pm 0.02$ & [45] \\
\hline
\end{tabular}

would not be enough to produce significant deviations from the time-temperature superposition principle but could induce a slight acceleration of the dynamics with respect to the ideal behavior in this temperature range, leading to an apparent lower value of $T_{c}$.

\section{DISCUSSION}

We have obtained very consistent results in this phenomenological approach to the application of MCT to PIB simulation data. We recall that this was also the main conclusion of the experimental work by Kisliuk et al. [37] when the theory was applied to light scattering PIB data in the high temperature range. It is also important to note that the $b$-exponent resulting from our analysis is compatible with the range reported in that work (see Table I), if we consider the uncertainties in its determination (see the shadowed area in Fig. 5). Moreover, like from the light scattering study, we also obtain an unusually high value of $T_{c}$ as compared with other systems (see below). Thus, experimental and simulation works point to the same range of values for the relevant MCT parameters of PIB (exponent parameters and $T_{c}$ ).

A first question one could ask is what is the influence of considering other kinds of atoms for the self-correlation function in the MCT analysis. In the particular case of $\mathrm{PIB}$, it is clear that the methyl groups undergo additional dynamic processes with respect to the main-chain atoms above considered. We have thus checked the factorization theorem and the TTSP with the methyl-group hydrogens $(\mathrm{mH})$ in the system. The results are presented in Fig. 11. The MCT $\beta$-regime in this case is apparently reduced - the factorization theorem fails at about 100 ps instead of 200 ps. To look at the origin of this effect we consider the results of the analysis of methyl group rotations in PIB obtained from these simulations and published elsewhere [55]. In that work, the relative motion of methyl-group hydrogens with respect to the carbon to which they are linked has been characterized in the spirit of the rotational rate distribution model $[58,59]$. Such model introduces the ingredient of the disorder inherent to glassy materials assuming a log-Gaussian distribution of rotational rates (or equivalently, rotational times $\tau_{R}$ ) for methyl groups. In Fig. 11(a) we have included as circles the distribution function obtained for $\tau_{R}$ of PIB in Ref. [55] for the considered temperature. As illustrated in this figure, in the temperature range of applicability of the MCT the characteristic times for methyl group rotations show distribution functions centered just in the time interval of the von Schweidler regime [55]. Furthermore, the factorization in real space reveals that the localization of the $\mathrm{mH}$ motions is much less severe than that of the main-chain carbons [Fig. 11(b) vs Fig. 3]. Regarding the TTSP [Fig. 11(c)], a good superposition is observed only in the very last part of the decay (times similar or longer than that where the self-correlation function has decayed to about 0.2 of the initial value -above defined as $\tau$ ). The methyl group rotation thus strongly influences the dynamics of this kind of atoms in the time region between the $\beta$ and $\alpha$-processes in this polymer. On the other hand, the KWW analysis of the self-correlation function of methyl-group hydrogens delivers $\beta$-values that in general are lower than those obtained for main-chain carbons, as can be seen in Fig. 12. Consequently, the value deduced for the $b$-exponent would also be lower, namely $b=0.25$, leading to $a=0.18, \lambda=0.93$ and $\gamma=4.70$. A fit of Eq. (11) to the corresponding characteristic times $\tau$ as defined above for $\mathrm{mHs}$ and imposing such $\gamma$-value results in a critical temperature of about $260 \mathrm{~K}$, i.e., $30-50 \mathrm{~K}$ below that deduced for main-chain carbons. The reported experimental value of $T_{c}$ is in the range $270-280 \mathrm{~K}$, i.e., between the two values deduced from the simulations. Finally, we note that considering the main-chain hydrogens the $b$-value deduced is the same as that obtained for the main-chain carbons (see Fig. 12).

Another point worth of discussion concerns the localization length $r_{s c}$. From the Gaussian fit of $f_{Q}^{s c}$ we have found $r_{s c}=0.3 \AA$ for main-chain carbons. Conversely, from the 

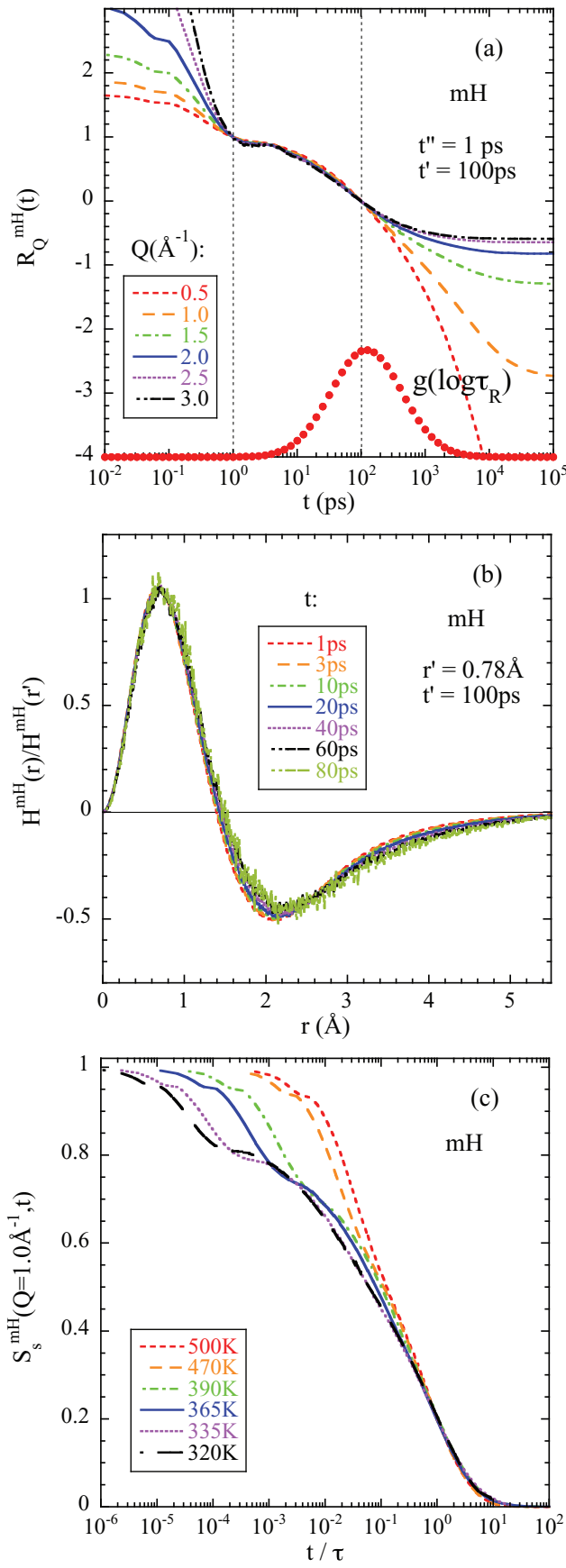

FIG. 11. (Color online) Test of the factorization theorem in reciprocal (a) and real space (b) and of the TTSP (c) applied to the self-correlation function of the methyl-group hydrogens at $335 \mathrm{~K}$. The circles in (a) reproduce the Gaussian distribution of rotational times obtained for PIB in Ref. [55] at this temperature.

factorization theorem a value of $0.92 \AA$ has been obtained for $r_{o}$, i.e., the distance at which the function $H(r)$ vanishes. Assuming the Gaussian approximation,

$$
\frac{H(r)}{H\left(r^{\prime}\right)} \approx \frac{\left(1-r^{2} / 6 r_{s c}^{2}\right) \exp \left(-r^{2} / 4 r_{s c}^{2}\right)}{\left(1-r^{\prime 2} / 6 r_{s c}^{2}\right) \exp \left(-r^{2} / 4 r_{s c}^{\prime 2}\right)},
$$

$r_{o}$ should be given by $\sqrt{6} r_{s c}=0.73 \AA$. The slightly larger value obtained for $r_{o}$ indicates actual deviations of the selfpart of the van Hove correlation function from the Gaussian

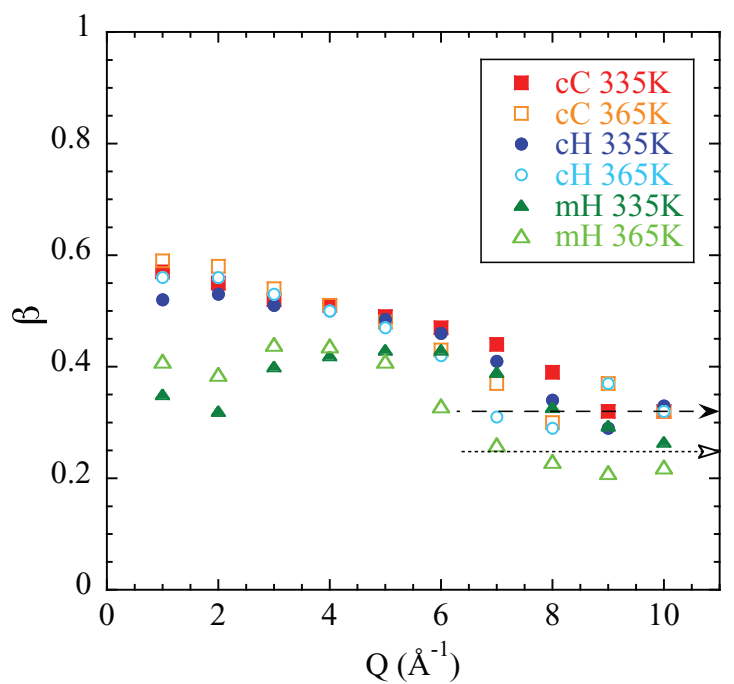

FIG. 12. (Color online) $Q$-dependence of the stretching parameter $\beta$ evaluated at $335 \mathrm{~K}$ (solid) and $365 \mathrm{~K}$ (empty) of the self-correlation function of main-chain carbons (squares), main-chain hydrogens (circles), and methyl-group hydrogens (triangles). Dashed arrow marks the average asymptotic value for main-chain atoms and dotted arrow that for methyl-group hydrogens.

approximation in the $\beta$-regime. These can be quantified by the second order non-Gaussian parameter $\alpha_{2}(t)$ defined as

$$
\alpha_{2}(t)=\frac{3}{5} \frac{\left\langle r^{4}(t)\right\rangle}{\left\langle r^{2}(t)\right\rangle^{2}}-1,
$$

where $\left\langle r^{2 n}\right\rangle$ are moments of the self-part of the van Hove correlation function $G_{s}(r, t)$. As can be seen in Fig. 13, this parameter takes non-negligible values in the time region of the $\beta$-process. On the other hand, taking into account that the interchain distance $d_{\text {chain }}$ in PIB is $6.3 \AA, r_{s c} \approx 0.05 d_{\text {chain. }}$. This value is half of the value corresponding to the Lindemann criterion of melting. This criterion states that a (crystalline) solid melts if the particle displacements about the equilibrium

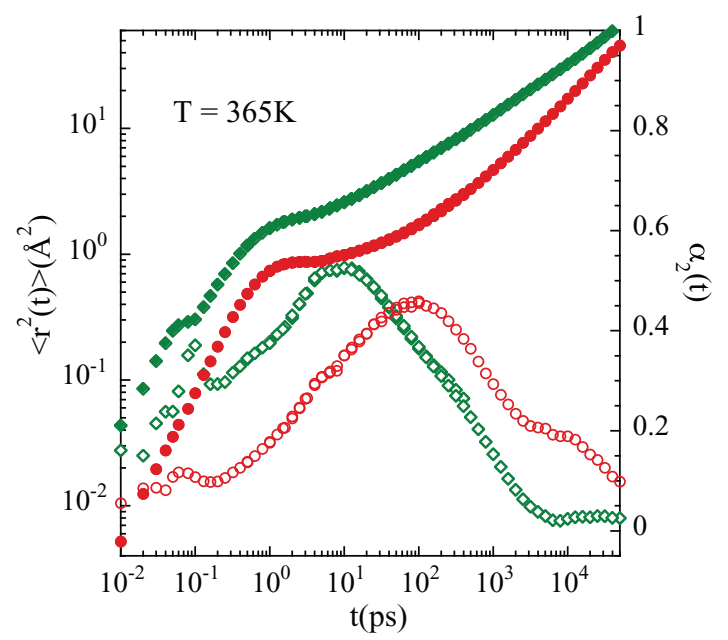

FIG. 13. (Color online) Mean squared displacement (filled symbols) and non-Gaussian parameter (empty symbols) of main-chain carbons (circles) and methyl-group hydrogens (diamonds) calculated for PIB at $365 \mathrm{~K}$. 
TABLE II. Values of the localization length $r_{s c}$, the interchain distance $d_{\text {chain }}$, the ratio between $T_{c}$ and $T_{g}$ and the fragility $m$ for different polymers. The references for $r_{s c}$ and $T_{c} / T_{g}$ are the respective ones listed in Table I.

\begin{tabular}{lclccc}
\hline \hline Polymer & $r_{s c}(\AA)$ (main-chain carbons) & $r_{s c}(\AA)($ hydrogens) & $d_{\text {chain }}(\AA)$ & $T_{c} / T_{g}$ & $m$ \\
\hline Polyisobutylene & 0.30 & 0.60 (methyl-group) & 6.3 & 1.5 & $46[66,67]$ \\
1,4-Polybutadiene & & 0.30 & 4.5 & 1.17 & $97[68], 107[69]$ \\
Poly(vinyl methyl ether) & 0.28 & 0.47 (main-chain) & 6.3 & 1.2 & $81[70], 75[66]$ \\
Polystyrene (exp.) & & & 1.15 & $116[71], 139[66], 143[72]$ \\
Poly(propylene glycol) (exp.) & & & 1.18 & $75[66,73], 71[45], 104[45]$ \\
\hline \hline
\end{tabular}

position reach about $10 \%$ of the particle diameter [64]. In beadspring polymer models [19] it was shown that the localization length is very close to the Lindemann criterion. In the case of real polymers, we have to note that the localization length depends on the kind of atom considered. For example, for the methyl-group hydrogens in PIB we had already commented a larger localization length than for main-chain carbons from simple inspection of Fig. 11(b). This can also be appreciated in the mean squared displacements represented in Fig. 13. Considering the Gaussian approximation and the $r_{o}$ position, a value of $0.6 \AA$ is deduced for $r_{s c}$ of methyl-group hydrogens (twice that of main-chain carbons). This value would be much closer to satisfy the Lindemann criterion. Regarding other polymers, the values reported for $r_{s c}$ in the literature are listed in Table II. Apparently, the criterion is roughly fulfilled only for the hydrogens.

Specially worth of discussion is the observation of a clearly higher value of the exponent parameter $\lambda=0.89$ here found for PIB as compared to other glass-forming systems $[14,60,62,63]$. Low-molecular weight systems, as $o$-terphenyl, silica or water, show values similar to that of hard spheres $(\lambda=0.74)$. We note that in a similar way as for PIB, rather high $\lambda$-values have recently been reported for fully atomistic $1,4-\mathrm{PB}$ [24] and PVME [25] ( $\lambda=0.93$ and 0.87, respectively). In those works, such finding was attributed to the local conformational changes controlled by rotational barriers, that affect the dynamics of the polymer segments within the cage. It is worth noting that chain connectivity in fully flexible bead-spring models does not yield significant changes in $\lambda$. In fact, in a recent work on MD simulations of a simple model for polymer melts with intramolecular barriers [27] it has been found that there is a clear correlation between the exponent parameter and chain stiffness. In their interpretation, the competition between packing effects and intramolecular barriers combined with chain connectivity is the mechanism responsible for the increase of $\lambda$ above $\lambda \approx 0.76$ (corresponding to fully flexible chains), when torsional and bending potentials are included in the simulated bead-spring model. In that work, the effect of these intramolecular barriers was characterized by the local stiffness of the chain, measured by the well-known "characteristic ratio $C_{n}$ " introduced by Flory [65]. Here $n$ is the number of main-chain bonds. Values of $\lambda \approx 0.9$ were obtained for very stiff chains (chains of 10 beads with $C_{10} \approx 4.5$ ). Chemically realistic polymer models seem to exhibit $\lambda$-values approaching the limit $\lambda=1$. For example, a united atom model of polyethylene [21] reveals $\lambda=0.81$; for $1,4-\mathrm{PB}$, values of 0.90 and 0.93 have been reported from a united atom model as well [22] and a fully atomistic model [24], respectively.
The characteristic ratios in these cases are above 4 . We note that from MCT analysis of experimental data (1,4-PB, PIB, PS and PPG) also rather small values of the $b$-parameter (and consequently large values for the exponent parameter $\lambda$ ) have been reported, as can be seen in Table I. Again, such real polymers show relatively large characteristic ratios. For our simulated PIB the characteristic ratio $C_{n}$ has been calculated from the average of the squared end-to-end vector distance $\left\langle R_{e}^{2}\right\rangle$ as $C_{n}=\left\langle R_{e}^{2}\right\rangle /\left(n \ell^{2}\right)$ ( $n$ : number of $\mathrm{C}-\mathrm{C}$ bonds along the main chain, $\ell=1.54 \AA$ : $\mathrm{C}-\mathrm{C}$ bond length), leading to a value of $5.5 \pm 0.5$. Thus, these PIB simulations perfectly fit in this tendency. We can then conclude that the previously mentioned studies and the present one strongly support the scenario proposed in [27], where real polymers would be classified in the family of complex systems - as short-ranged attractive colloids [74-76] or binary mixtures with strong dynamic asymmetry [77-80]—which seem to show two competing mechanisms of freezing. In the case of polymers these two mechanisms would be the intermolecular packing and the intramolecular barriers for conformational changes combined with chain connectivity. The unusually large $\lambda$-values exhibited by polymers could reveal an underlying high-order MCT transition. We note, however, that the typical precursor effects characterizing nearby MCT high-order transitions-as, for instance, the convex-to-concave crossover exhibited by the density-density correlators-are not observed in the case of PIB simulations and in polymers in general. This might suggest that the distance in the explored control parameter space to the high-order transition is still rather large.

It is worthy of remark that the idea of two different mechanisms contributing to the glass-transition in polymers is not new in the field of polymer science. For instance, from a phenomenological point of view it has usually been considered that the experimental values of $T_{g}$ depend crucially upon two important factors: chain stiffness or flexibility and intermolecular interactions. By considering the values of the characteristic ratio (chain stiffness or flexibility) and the density of cohesive energy (intermolecular interactions) of many polymers, some empirical rules have also been proposed more than 20 years ago (see, e.g., [81]). This question has also been recently revisited in terms of the entropy theory of glass-formation in polymers [82]. The results reported here and in the previous works above mentioned [24,25,27] suggest that MCT might be a suitable theoretical framework to address this question. However, whether or not the increase of chain stiffness at constant density and temperature would produce all the signatures characteristic of a glass-transitionlike phenomenon is a question that demands for further work. 


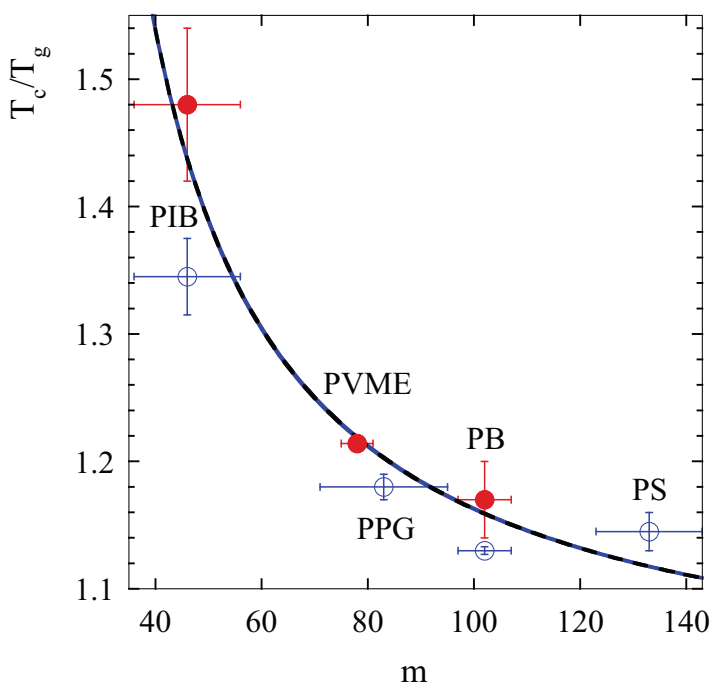

FIG. 14. (Color online) Ratio between the critical temperature and the glass-transition temperature as function of the fragility index for PIB and the different polymers available in the literature. Solid points indicate that the result arises from MD-simulations studies, while empty symbols are deduced from experimental investigations. Line corresponds to the law $T_{c} / T_{g}=m /(m-b)$ with $b=14$ as proposed in Ref. [84].

Finally, we comment on the salient result of the MCT analysis of PIB regarding the value of $T_{c}$. The obtained value is very high with respect to that usually found $\left(T_{c} \approx 1.2 T_{g}\right)$ not only in low-molecular weight glass-forming systems but also for polymers (see the compilation in Table II). This observation was attributed in Ref. [37] to the also particularly strong character (in Angell's classification based on the concept of fragility [43]) of PIB. As shown in Ref. [83], the $T_{c} / T_{g}$ ratio tends to decrease with the fragility of the system for lowmolecular glass-forming systems. To check whether this could also be the case for polymers, we have represented in Fig. 14 the available values of the $T_{c} / T_{g}$ ratio for polymers as function of the corresponding reported fragility parameters, defined as

$$
m=\left.\frac{\partial \log \tau_{\alpha}}{\partial\left(T_{g} / T\right)}\right|_{T=T_{g}} .
$$

Taking into account the large uncertainties usually involved in determining the value of the fragility index, the proposed trend also seems to work in glass-forming polymers. In fact, it seems that the data available nicely follow an analytical law relating the $T_{c} / T_{g}$ ratio and $m$ (see the solid line in Fig. 14) which was recently proposed by Saltzman and Schweizer [84]. Then, the temperature difference between $T_{c}$ and $T_{g}$ would reflect the fragility of polymeric liquids.

\section{CONCLUSIONS}

The applicability of Mode Coupling Theory to PIB has been explored. We have obtained consistent results in the application of MCT phenomenological predictions to the dynamic structure factor and the self-correlation function of PIB main-chain carbons calculated from our simulation data. Considering the self-correlation function of methyl-group hydrogens the analysis is hampered by the occurrence of the specific methyl group rotations with characteristic times in the von Schweidler regime. On the other hand, a larger localization length $r_{s c}$ is deduced for methyl-group hydrogens with respect to that of main-chain carbons. Results reported for other polymers also indicate larger values of $r_{s c}$ for hydrogens than for main-chain carbons; the Lindemann criterion would be satisfied only by the former subspecies.

The values of the exponent parameter and critical temperature obtained from our MCT analysis of PIB agree, within the uncertainties involved in both studies, with those deduced from depolarized light scattering experiments [37]. Both, $\lambda$ and $T_{c} / T_{g}$ values found for PIB are unusually large with respect to those usually obtained in low molecular weight systems. The high $T_{c} / T_{g}$ value is compatible with a certain correlation of this parameter with the fragility in Angell's classification, as proposed by Sokolov [83] and more recently by Saltzman and Schweizer [84]. Conversely, the value of $\lambda$ is close to that reported for real polymers like 1,4-PB [18], PPG [45], or PS [37], simulated polymers like 1,4-PB [24] and PVME [25], and simple polymer models with intramolecular barriers [27]. Such finding could be interpreted in the framework of a higher-order MCT transition for real polymers arising from the simultaneous occurrence of two mechanisms leading to dynamic arrest: (i) packing, of intermolecular character and present in all glass-forming systems; (ii) barriers for conformational changes, of intramolecular origin, which combined with chain connectivity are specific of macromolecular systems [24,27]. Our findings in PIB seem to confirm the generality of this framework.

\section{ACKNOWLEDGMENTS}

Y.K. acknowledges the grant of the Spanish Ministry. We thank support by the projects no. IT-654-13 (GV) and MAT2012-31088.
[1] W. Götze and L. Sjögren, Rep. Prog. Phys. 55, 241 (1992).

[2] W. Götze, in Liquids, Freezing, Glass Transition, edited by J. P. Hansen, D. Levesque, and J. Zinn-Justin (North-Holland, Amsterdam, 1991).

[3] W. Götze, Complex Dynamics of Glass-Forming Liquids: A Mode Coupling Theory (Oxford University Press, New York, 2009).
[4] W. Götze, J. Phys.: Condens. Matter 11, A1 (1999).

[5] D. R. Reichmann and P. Charbonneau, J. Stat. Mech. (2005) P05013.

[6] S. P. Das, Rev. Mod. Phys. 76, 785 (2004).

[7] W. Götze and L. Sjögren, Phys. Rev. A 43, 5442 (1991).

[8] G. Foffi, W. Götze, F. Sciortino, P. Tartaglia, and T. Voigtmann, Phys. Rev. E 69, 011505 (2004). 
[9] J.-L. Barrat and A. Latz, J. Phys.: Condens. Matter 2, 4289 (1990).

[10] M. Nauroth and W. Kob, Phys. Rev. E 55, 657 (1997).

[11] S. H. Chong, W. Götze, and A. P. Singh, Phys. Rev. E 63, 011206 (2000).

[12] R. Schilling and T. Scheidsteger, Phys. Rev. E 56, 2932 (1997).

[13] L. Fabbian, A. Latz, R. Schilling, F. Sciortino, P. Tartaglia, and C. Theis, Phys. Rev. E 62, 2388 (2000).

[14] T. Franosch, M. Fuchs, W. Götze, M. R. Mayr, and A. P. Singh, Phys. Rev. E 56, 5659 (1997).

[15] J. Wuttke, J. Hernandez, G. Li, G. Coddens, H. Z. Cummins, F. Fujara, W. Petry, and H. Sillescu, Phys. Rev. Lett. 72, 3052 (1994).

[16] A. Tölle, H. Schober, J. Wuttke, and F. Fujara, Phys. Rev. E 56, 809 (1997).

[17] D. Richter, B. Frick, and B. Farago, Phys. Rev. Lett. 61, 2465 (1988).

[18] R. Zorn, D. Richter, B. Frick, and B. Farago, Physica A 201, 52 (1993).

[19] J. Baschnagel and F. Varnik, J. Phys.: Condens. Matter 17, R851 (2005).

[20] S.-H. Chong and M. Fuchs, Phys. Rev. Lett. 88, 185702 (2002).

[21] A. van Zon and S. W. de Leeuw, Phys. Rev. E 60, 6942 (1999).

[22] W. Paul, D. Bedrov, and G. D. Smith, Phys. Rev. E 74, 021501 (2006).

[23] S.-H. Chong, M. Aichele, H. Meyer, M. Fuchs, and J. Baschnagel, Phys. Rev. E 76, 051806 (2007).

[24] J. Colmenero, A. Narros, F. Alvarez, A. Arbe, and A. J. Moreno, J. Phys.: Condens. Matter 19, 205127 (2007)

[25] S. Capponi, A. Arbe, F. Alvarez, J. Colmenero, B. Frick, and J. P. Embs, J. Chem. Phys. 131, 204901 (2009).

[26] W. Paul and G. D. Smith, Rep. Prog. Phys. 67, 1117 (2004).

[27] M. Bernabei, A. J. Moreno, and J. Colmenero, Phys. Rev. Lett. 101, 255701 (2008).

[28] W. P. Slichter, J. Polym. Sci.: Part C 14, 33 (1966).

[29] J. D. Ferry, Viscoelastic Properties of Polymers (John Wiley \& Sons, New York, 1970).

[30] P. J. Törmälä, Macromol. Sci. Rev. Macromol. Chem. C 17, 297 (1979).

[31] R. Dejean de la Batie, F. Lauprtre, and L. Monnerie, Macromolecules 22, 2617 (1989).

[32] B. Frick, D. Richter, and S. Trevino, Physica A 201, 88 (1993).

[33] B. Frick and D. Richter, Phys. Rev. B 47, 14795 (1993).

[34] D. Richter, A. Arbe, J. Colmenero, M. Monkenbusch, B. Farago, and R. Faust, Macromolecules 31, 1133 (1998).

[35] A. Arbe, J. Colmenero, B. Frick, M. Monkenbusch, and D. Richter, Macromolecules 31, 4926 (1998).

[36] D. Richter, M. Monkenbusch, J. Allgaier, A. Arbe, J. Colmenero, B. Farago, Y. Cheol Bae, and R. Faust, J. Chem. Phys. 111, 6107 (1999).

[37] A. Kisliuk, R. T. Mathers, and A. P. Sokolov, J. Polym. Sci., Part B: Polym. Phys. 38, 2785 (2000).

[38] A. Arbe, M. Monkenbusch, J. Stellbrink, D. Richter, B. Farago, K. Almdal, and R. Faust, Macromolecules 34, 1281 (2001).

[39] B. Farago, A. Arbe, J. Colmenero, R. Faust, U. Buchenau, and D. Richter, Phys. Rev. E 65, 051803 (2002).
[40] V. Arrighi, A. Triolo, and H. Qian, J. Non-Cryst. Solids 307-310, 654 (2002).

[41] A. Arbe, J. Colmenero, B. Farago, M. Monkenbusch, U. Buchenau, and D. Richter, Chem. Phys. 292, 295 (2003).

[42] M. A. Adams, B. J. Gabrys, W. M. Zajac, and D. G. Peiffer, Macromolecules 38, 160 (2005).

[43] C. A. Angell, Science 267, 1924 (1995).

[44] A. P. Sokolov, W. Steffen, and E. Rössler, Phys. Rev. E 52, 5105 (1995).

[45] R. Bergman, L. Borjesson, L. M. Torell, and A. Fontana, Phys. Rev. B 56, 11619 (1997).

[46] T. Franosch, W. Götze, M. R. Mayr, and A. P. Singh, Phys. Rev. E 55, 3183 (1997).

[47] S. W. Bunte and H. Sun, J. Phys. Chem. B 104, 2477 (2000).

[48] H. Sun, J. Phys. Chem. B 102, 7338 (1998).

[49] J. Yang, Y. Ren, A. Tian, and H. Sun, J. Phys. Chem. B 104, 4951 (2000).

[50] D. N. Theodorou and U. W. Suter, Macromolecules 18, 1467 (1985).

[51] L. J. Fetters, D. J. Lohse, D. Richter, T. A. Witten, and A. Zirkel, Macromolecules 27, 4639 (1994).

[52] Physical Properties of Polymers Handbook, edited by J. E. Mark (Springer, New York, 1996).

[53] J. Colmenero, F. Alvarez, and A. Arbe, Phys. Rev. E 65, 041804 (2002).

[54] Y. Khairy, F. Alvarez, A. Arbe, and J. Colmenero [Macromolecules (to be published)].

[55] Y. Khairy, F. Alvarez, A. Arbe, and J. Colmenero [Revisiting Previous Neutron Scattering Studies (to be published)].

[56] B. Doliwa and A. Heuer, Phys. Rev. Lett. 80, 4915 (1998).

[57] M. Fuchs, J. Non-Cryst. Solids 172, 241 (1994).

[58] A. Chahid, A. Alegría, and J. Colmenero, Macromolecules 27, 3282 (1994).

[59] J. Colmenero, A. J. Moreno, and A. Alegría, Prog. Polym. Sci. 30, 1147 (2005).

[60] S. Mossa, R. Di Leonardo, G. Ruocco, and M. Sampoli, Phys. Rev. E 62, 612 (2000).

[61] W. Petry, E. Bartsch, F. Fujara, M. Kiebel, H. Sillescu, and B. Farago, Z. Phys. B 83, 175 (1991).

[62] J. Horbach and W. Kob, Phys. Rev. E 64, 041503 (2001).

[63] F. Sciortino, L. Fabbian, S.-H. Chen, and P. Tartaglia, Phys. Rev. E 56, 5397 (1997).

[64] F. H. Stillinger, Science 267, 1935 (1995).

[65] P. J. Flory, Statistical Mechanics of Chain Molecules (Interscience, New York, 1969).

[66] Q. Qin and G. B. McKenna, J. Non-Cryst. Solids 352, 2977 (2006).

[67] K. Kunal, M. Paluch, C. M. Roland, J. E. Puskas, Y. Chen, and A. P. Sokolov, J. Polym. Sci., Part B: Polym. Phys. 46, 1390 (2008).

[68] C. G. Robertson and C. M. Roland, Macromolecules 33, 1262 (2000).

[69] R. Zorn, G. B. McKenna, L. Willner, and D. Richter, Macromolecules 28, 8552 (1995).

[70] R. Casalini and C. M. Roland, J. Chem. Phys. 119, 4052 (2003).

[71] D. Huang and G. B. McKenna, J. Chem. Phys. 114, 5621 (2001).

[72] C. M. Roland, P. G. Santangelo, and K. L. Ngai, J. Chem. Phys. 111, 5593 (1999).

[73] R. Böhmer, K. L. Ngai, C. A. Angell, and D. J. Plazek, J. Chem. Phys. 99, 4201 (1993). 
[74] F. Sciortino, P. Tartaglia, and E. Zaccarelli, Phys. Rev. Lett. 91, 268301 (2003).

[75] M. Sperl, Phys. Rev. E 68, 031405 (2003).

[76] E. Zaccarelli, G. Foffi, K. A. Dawson, S. V. Buldyrev, F. Sciortino, and P. Tartaglia, Phys. Rev. E 66, 041402 (2002).

[77] A. J. Moreno and J. Colmenero, J. Chem. Phys. 124, 184906 (2006).

[78] A. J. Moreno and J. Colmenero, Phys. Rev. E 74, 021409 (2006).
[79] A. J. Moreno and J. Colmenero, J. Chem. Phys. 125, 164507 (2006).

[80] V. Krakoviack, Phys. Rev. Lett. 94, 065703 (2005).

[81] K. K. Chee, J. Appl. Polym. Sci. 43, 1205 (1991).

[82] E. B. Stukalin, J. F. Douglas, and K. F. Freed, J. Chem. Phys. 131, 114905 (2009).

[83] A. P. Sokolov, J. Non-Cryst. Solids 235-237, 190 (1998).

[84] E. J. Saltzman and K. S. Schweizer, J. Phys.: Condens. Matter 19, 205123 (2007). 